\title{
Gradient Estimates for a Nonlinear Heat Equation Under Finsler-geometric Flow
}

\author{
ZENG Fanqi* \\ School of Mathematics and Statistics, Xinyang Normal University, Xinyang 464000, \\ China.
}

Received 22 December 2019; Accepted 23 January 2020

\begin{abstract}
This paper considers a compact Finsler manifold $\left(M^{n}, F(t), m\right)$ evolving under a Finsler-geometric flow and establishes global gradient estimates for positive solutions of the following nonlinear heat equation

$$
\partial_{t} u(x, t)=\Delta_{m} u(x, t), \quad(x, t) \in M \times[0, T],
$$

where $\Delta_{m}$ is the Finsler-Laplacian. By integrating the gradient estimates, we derive the corresponding Harnack inequalities. Our results generalize and correct the work of S. Lakzian, who established similar results for the Finsler-Ricci flow. Our results are also natural extension of similar results on Riemannian-geometric flow, previously studied by J. Sun. Finally, we give an application to the Finsler-Yamabe flow.
\end{abstract}

AMS Subject Classifications: 53C44, 58J35, 53B40, 35K55

Chinese Library Classifications: O175.26

Key Words: Gradient estimate; nonlinear heat equation; Harnack inequality; Akbarzadeh's Ricci tensor; Finsler-geometric flow.

\section{Introduction}

The paper studies nonlinear heat equation

$$
\partial_{t} u(x, t)=\Delta_{m} u(x, t)
$$

on a compact Finsler manifold $\left(M^{n}, F(t), m\right)$ evolving by the Finsler-geometric flow

$$
\frac{\partial}{\partial t} g(t)=2 h(t)
$$

*Corresponding author. Email address: fanzeng10@126.com (F. Q. Zeng) 
where $(x, t) \in M \times[0, T], g(t)$ is the symmetric metric tensor associated with $F$, and $h(t)$ is a symmetric $(0,2)$-tensor field on $\left(M^{n}, F(t), m\right)$. An important example would be the case where $h(t)=-\operatorname{Ric}_{i j}(t)$ and $g(t)$ is a solution of the Finsler-Ricci flow introduced by Bao [1]. Unlike the usual Laplacian, the Finsler-Laplacian $\Delta_{m}$ is a nonlinear operator. For the existence, uniqueness and Sobolev regularity of a positive global solution of the nonlinear heat equation (1.1) (in the sense of distributions), we can see [2]. We will give some gradient estimates and Harnack inequalities for positive global solutions of equation (1.1).

The study of gradient estimates for the heat equation originated with the work of P. Li and S.-T. Yau [3]. They proved a space-time gradient estimate for positive solutions of the heat equation on a complete manifold. By integrating the gradient estimate along a space-time path, a Harnack inequality was derived. Therefore, Li-Yau inequality is often called differential Harnack inequality. Li-Yau type gradient estimates have been obtained for other nonlinear equations on manifolds, see for example [4-13] and the references therein. Over the past two decades, many authors used similar techniques to prove gradient estimates and Harnack inequalities for geometric flows. For instance, in [14], weakening Guenther's curvature constrains in [15] on the boundedness of the gradient of scalar curvature in addition to the boundedness of the Ricci curvature, Liu established first order gradient estimates for positive solutions of the heat equations on complete noncompact or closed Riemannian manifolds under Ricci flows. As applications, he derived Harnack type inequalities and second order gradient estimates for positive solutions. Generalizing Liu's work to general geometric flow, Sun [16] established first order and second order gradient estimates for positive solutions of the heat equations under general Riemannian-geometric flows. The list of relevant references includes but is not limited to [17-21].

Comparatively, there are less works on Finsler manifolds about gradient estimates of the nonlinear heat equation (1.1). To the best of our knowledge, in [22], Ohta and Sturm derived a Li-Yau gradient estimate as well as parabolic Harnack inequalities on compact Finsler manifolds. In [23], Lakzian derived differential Harnack estimates for positive global solutions to (1.1) under Finsler-Ricci flow. Later, the author and He [24] generalized and corrected Lakzian's results under some curvature constraints. Compared to the Riemannian case, it is harder to get the gradient estimate due to some obstructions. First, the solutions of (1.1) are lack of higher order regularity. Second, $\Delta_{m} u$ has no definition at the maximum point of $u$, and thus we cannot use Finsler-Laplacian to adopt maximum principle. Last but not least, in view of nonlinear property of gradient operator, it is difficult to do the calculations. In this paper, we follow the work of Sun [16], and establish some gradient estimates for positive global solutions of (1.1) under Finsler-geometric flow (1.2), which are richer than [16,22-24].

The rest of this paper is organized as follows.

In Section 2, we first briefly review some facts and results about Finsler geometry. In Section 3, we establish space-time gradient estimates for positive global solution of (1.1), 
see Theorem 3.1. We also give the corresponding Harnack inequalities, see Corollary 3.1. Finally, in Section 4, we give two applications to the Finsler-Ricci flow and FinslerYamabe flow.

\section{Preliminaries}

In this section we briefly recall the fundamentals of Finsler geometry by Bao, Chern and Shen [25], as well as some results on the analysis of Finsler geometry by Ohta-Sturm $[2,22]$.

\subsection{Finsler metric}

We assume that $M$ is an $n$-dimensional smooth connected manifold. Let TM be the tangent bundle over $M$ with local coordinates $(x, y)$, where $x=\left(x^{1}, \cdots, x^{n}\right)$ and $y=\left(y^{1}, \cdots, y^{n}\right)$. A Finsler metric on $M$ is a function $F: T M \longrightarrow[0, \infty)$ satisfying the following properties:

(i) F is smooth on $T M \backslash\{0\}$;

(ii) $F(x, \lambda y)=\lambda F(x, y)$ for all $\lambda>0$;

(iii) For any nonzero tangent vector $y \in T M$, the approximated symmetric metric tensor, $g_{y}$, defined by

$$
g_{y}(u, v):=\left.\frac{1}{2} \frac{\partial^{2}}{\partial s \partial t} F^{2}(y+s u+t v)\right|_{s=t=0^{\prime}}
$$

is positive definite.

Such a pair $\left(M^{n}, F\right)$ is called a Finsler manifold. A Finsler structure is said to be reversible if, in addition, $F$ is even. Otherwise $F$ is nonreversible. We say a Finsler manifold $\left(M^{n}, F\right)$ is forward (resp.backward) complete if every geodesic defined on $[0, a]$ (resp. $[-a, 0])$ can be extended to $[0,+\infty)$ (resp. $(-\infty, 0])$. Compact Finsler manifolds are both forward and backward complete. By a Finsler measure space we mean a triple $\left(M^{n}, F, m\right)$ constituted with a smooth, connected $n$-dimensional manifold $M$, a Finsler structure $F$ on $M$ and a measure $m$ on $M$.

\subsection{Geodesic spray and Chern connection}

It is straightforward to observe that the geodesic spray in the Finsler setting is of the form, $G=y^{i} \frac{\partial}{\partial x^{i}}-2 G^{i}(x, y) \frac{\partial}{\partial y^{i}}$ where,

$$
G^{i}(x, y)=\frac{1}{4} g_{y}^{i k}\left\{2 \frac{\partial\left(g_{y}\right)_{j k}}{\partial x^{l}}-\frac{\partial\left(g_{y}\right)_{j l}}{\partial x^{k}}\right\} y^{j} y^{l}
$$


For every nonvanishing vector field $V, g_{i j}(V)$ induces a Riemannian structure $g_{V}$ of $T_{x} M$ via

$$
g_{V}(X, Y)=\sum_{i, j}^{n} g_{i j}(V) X^{i} Y^{j}, \quad \text { for } X, Y \in T_{X} M .
$$

In particular, $g_{V}(V, V)=F^{2}(V)$.

The projection $\pi: T M \longrightarrow M$ gives rise to the pull-back bundle $\pi^{*} T M$ over $T M \backslash\{0\}$. As is well known, on $\pi^{*} T M$ there exists uniquely the Chern connection $D$. The Chern connection is determined by the following structure equations, which characterize "torsion freeness":

$$
D_{X}^{V} Y-D_{Y}^{V} X=[X, Y]
$$

and "almost g-compatibility"

$$
Z\left(g_{V}(X, Y)\right)=g_{V}\left(D_{Z}^{V} X, Y\right)+g_{V}\left(X, D_{Z}^{V} Y\right)+C_{V}\left(D_{Z}^{V} V, X, Y\right)
$$

for $V \in T M \backslash\{0\}, X, Y, Z \in T M$. Here

$$
C_{V}(X, Y, Z)=\frac{1}{4} \frac{\partial^{3} F^{2}}{\partial V^{i} \partial V^{j} \partial V^{k}}(V) X^{i} Y^{j} Z^{k}
$$

denotes the Cartan tensor and $D_{X}^{V} Y$ the covariant derivative with respect to reference vector $V \in T M \backslash\{0\}$. We mention here that $C_{V}(V, X, Y)=0$ due to the homogeneity of $F$. The Chern connection coefficients are given by

$$
\Gamma_{j k}^{i}:=\frac{1}{2} g^{i l}\left\{\frac{\partial g_{l j}}{\partial x^{k}}-\frac{\partial g_{j k}}{\partial x^{l}}+\frac{\partial g_{k l}}{\partial x^{j}}-\frac{\partial g_{l j}}{\partial y^{r}} N_{k}^{r}+\frac{\partial g_{j k}}{\partial y^{r}} N_{l}^{r}-\frac{\partial g_{k l}}{\partial y^{r}} N_{j}^{r}\right\},
$$

where $N_{j}^{i}=\partial G^{i} / \partial y^{j}$ and $g$ is in fact $g_{y}$.

\subsection{Covariant derivation of tensor field}

Given the coordinates $\left\{x^{i}, y^{i}\right\}$ on TM, one can observe that the pair $\left\{\frac{\delta}{\delta x^{i}}, \frac{\partial}{\partial y^{i}}\right\}$ forms a horizontal and vertical frame for TTM, where $\frac{\delta}{\delta x^{i}}=\frac{\partial}{\partial x^{i}}-N_{i}^{k} \frac{\partial}{\partial y^{k}}$. Let $\left\{d x^{i}, \delta y^{i}\right\}$ denote the local frame dual to $\left\{\frac{\delta}{\delta x^{i}}, \frac{\partial}{\partial y^{i}}\right\}$, where $\delta y^{i}=d y^{i}+N_{j}^{i} d x^{j}$. Then we obtain a decomposition for $T(T M \backslash\{0\})$ and $T^{*}(T M \backslash\{0\})$,

$$
T(T M \backslash\{0\})=\mathcal{H} T M \oplus \mathcal{V} T M, \quad T^{*}(T M \backslash\{0\})=\mathcal{H}^{*} T M \oplus \mathcal{V}^{*} T M,
$$

where

$$
\begin{aligned}
& \mathcal{H} T M=\operatorname{span}\left\{\frac{\delta}{\delta x^{i}}\right\}, \quad \mathcal{V} T M=\operatorname{span}\left\{\frac{\partial}{\partial y^{i}}\right\}, \\
& \mathcal{H}^{*} T M=\operatorname{span}\left\{d x^{i}\right\}, \quad \mathcal{V}^{*} T M=\operatorname{span}\left\{\delta y^{i}\right\} .
\end{aligned}
$$


Let $T=T^{i j} \frac{\partial}{\partial x^{i}} \otimes \frac{\partial}{\partial x^{j}}$ be an arbitrary smooth local section of $\pi^{*} T M \otimes \pi^{*} T^{*} M$. They can therefore be expanded in terms of the natural basis $\left\{d x^{s}, \frac{\delta y^{s}}{F}\right\}$. The covariant derivative of $T^{i j}$ denotes

$$
(\nabla T)^{i j}=T_{\mid s}^{i j} d x^{s}+T_{; s}^{i j} \frac{\delta y^{s}}{F} .
$$

The horizontal covariant derivative $T_{\mid s}^{i j}$ denotes

$$
T_{\mid s}^{i j}=\frac{\delta T^{i j}}{\delta x^{s}}+T^{k i} \Gamma_{k s}^{j}+T^{k j} \Gamma_{k s}^{i} .
$$

The vertical covariant derivative $T_{; s}^{i j}$ denotes

$$
T_{; s}^{i j}=F \frac{\partial T^{i j}}{\partial y^{s}} .
$$

\subsection{Distance function}

For $x_{1}, x_{2} \in M$, the distance function from $x_{1}$ to $x_{2}$ is defined by

$$
d\left(x_{1}, x_{2}\right)=\inf _{\gamma} \int_{0}^{1} F(\dot{\gamma}(t)) \mathrm{d} t
$$

where the infimum is taken over all $C^{1}$-curves $\gamma:[0,1] \rightarrow M$ such that $\gamma(0)=x_{1}$ and $\gamma(1)=$ $x_{2}$. Note that the distance function may not be symmetric unless $F$ is reversible. A $C^{\infty}$ curve $\gamma:[0,1] \rightarrow M$ is called a geodesic if $F(\dot{\gamma})$ is constant and it is locally minimizing. In terms of the Chern connection, a geodesic $\gamma$ satisfies $D_{\dot{\gamma}}^{\dot{\gamma}} \dot{\gamma}=0$.

\subsection{S-Curvature}

Associated to any Finsler structure, there is one canonical measure, called the BusemannHausdorff measure, given by

$$
\mathrm{d} V_{F}:=\sigma_{F}(x) \mathrm{d} x^{1} \wedge \cdots \wedge \mathrm{d} x^{n},
$$

where $\sigma_{F}(x)$ is the volume ratio

$$
\sigma_{F}(x)=\frac{\operatorname{vol}\left(B_{\mathbb{R}^{n}}(1)\right)}{\operatorname{vol}\left(\left(a_{i}\right) \in \mathbb{R}^{n} \mid F\left(\sum a_{i} \frac{\partial}{\partial x^{i}}\right)<1\right)} .
$$

The $S$-curvature is then defined as

$$
S(y):=\frac{\partial G^{i}}{\partial y^{i}}(x, y)-y^{i} \frac{\partial}{\partial x^{i}}\left(\ln \sigma_{F}(x)\right) .
$$




\subsection{Legendre transform, Gradient, Hessian and Finsler-Laplacian}

In order to define the gradient of a function, we define the Legendre transform $\mathcal{L}: T M \longrightarrow$ $T^{*} M$, as $\mathcal{L}(y)=F F_{y^{i}} d x^{i}$, which satisfies $\mathcal{L}(0)=0$ and $\mathcal{L}(\lambda y)=\lambda \mathcal{L}(y)$ for all $\lambda>0$ and $y \in T M \backslash\{0\}$. Then $\mathcal{L}: T M \backslash\{0\} \longrightarrow T^{*} M \backslash\{0\}$ is a norm-preserving $C^{\infty}$ diffeomorphism. For a smooth function $u: M \longrightarrow \mathbb{R}$, the gradient vector of $u$ at $x \in M$ is defined as $\nabla u(x):=$ $\mathcal{L}^{-1}(d u(x)) \in T_{x} M$, which can be written as

$$
\nabla u(x):= \begin{cases}g^{i j}(x, \nabla u) \frac{\partial u}{\partial x^{j}} \frac{\partial}{\partial x^{i}}, & \mathrm{~d} u(x) \neq 0 \\ 0, & \mathrm{~d} u(x)=0 .\end{cases}
$$

Set $M_{u}:=\{x \in M \mid d u(x) \neq 0\}$. We define $\nabla^{2} u(x) \in T_{x}^{*} M \otimes T_{x} M$ for $x \in M_{u}$ by using the following covariant derivative

$$
\nabla^{2} u(v):=D_{v}^{\nabla u} \nabla u(x) \in T_{x} M, \quad v \in T_{x} M .
$$

Set

$$
D^{2} u(X, Y):=g_{\nabla u}\left(\nabla^{2} u(X), Y\right)=g_{\nabla u}\left(D_{X}^{\nabla u}(\nabla u), Y\right) .
$$

Then we have

$$
g_{\nabla u}\left(D_{X}^{\nabla u}(\nabla u), Y\right)=D^{2} u(X, Y)=D^{2} u(Y, X)=g_{\nabla u}\left(D_{Y}^{\nabla u}(\nabla u), X\right),
$$

for any $X, Y \in T_{x} M$.

In order to define a Laplacian on Finsler manifolds, we need a measure $m$ (or a volume form $d m)$ on $M$. From now on, we consider the Finsler measure space $(M, F, m)$ equipped with a fixed smooth measure $m$. Let $V \in T M$ be a smooth vector field on $M$. In a local coordinate $\left(x^{i}\right)$, expressing $d m=e^{\Phi} d x^{1} d x^{2} \cdots d x^{n}$, we can write $\operatorname{div}_{m} V$ as

$$
\operatorname{div}_{m} V=\sum_{i=1}^{n}\left(\frac{\partial V^{i}}{\partial x^{i}}+V^{i} \frac{\partial \Phi}{\partial x^{i}}\right)
$$

A Laplacian, which is called the Finsler-Laplacian, can now be defined by

$$
\Delta_{m} u=\operatorname{div}_{m}(\nabla u) .
$$

We remark that the Finsler-Laplacian is better to be viewed in a weak sense that for $u \in$ $W^{1,2}(M)$,

$$
\int_{M} \phi \Delta_{m} u \mathrm{~d} m=-\int_{M} D \phi(\nabla u) \mathrm{d} m \quad \text { for } \quad \phi \in C_{c}^{\infty}(M),
$$

where $D \phi$ is the differential 1-form of $\phi$.

The relationship between $\Delta_{m} u$ and $\nabla^{2} u$ is that

$$
\Delta_{m} u=t r_{g_{\nabla u}}\left(\nabla^{2} u\right)-S(\nabla u)=\sum_{i=1}^{n} \nabla^{2} u\left(e_{i}, e_{i}\right)-S(\nabla u),
$$


where $\left\{e_{i}\right\}$ is an orthonormal basis of $T_{x} M$ with respect to $g_{\nabla u}$.

Given a vector field $V$, the weighted Laplacian is defined on the weighted Riemannian manifold $\left(M, g_{V}, m\right)$ by

$$
\Delta_{m}^{V} u=\operatorname{div}_{m}\left(\nabla^{V} u\right)
$$

where

$$
\nabla^{V} u(x):= \begin{cases}g^{i j}(x, V) \frac{\partial u}{\partial x^{j}} \frac{\partial}{\partial x^{i}}, & \mathrm{~d} u(x) \neq 0 \\ 0, & \mathrm{~d} u(x)=0 .\end{cases}
$$

Similarly, the weighted Laplacian can be viewed in a weak sense for $u \in W^{1,2}(M)$. We note that $\Delta_{m}^{\nabla u} u=\Delta_{m} u$.

\subsection{Weighted Ricci curvature}

The Ricci curvature of Finsler manifolds is defined as the trace of the flag curvature. Explicitly, given two linearly independent vectors $V, W \in T M \backslash\{0\}$, the flag curvature is defined by

$$
K^{V}(V, W)=\frac{g_{V}\left(R^{V}(V, W) W, V\right)}{g_{V}(V, V) g_{V}(W, W)-g_{V}(V, W)^{2}},
$$

where $R^{V}$ is the Chern curvature (or Riemannian curvature):

$$
R^{V}(X, Y) Z=D_{X}^{V} D_{Y}^{V} Z-D_{Y}^{V} D_{X}^{V} Z-D_{[X, Y]}^{V} Z
$$

Then the Ricci curvature is defined by

$$
\operatorname{Ric}(V)=\sum_{i=1}^{n-1} K^{V}\left(V, e_{i}\right),
$$

where $e_{1}, \cdots, e_{n-1}, \frac{V}{F(V)}$ form an orthonormal basis of $T_{x} M$ with respect to $g_{V}$.

We recall the definition of the weighted Ricci curvature on Finsler manifolds, which was introduced by Ohta in [26].

Given a vector $V \in T_{x} M$, let $\gamma:(-\varepsilon, \varepsilon) \rightarrow M$ be a geodesic with $\gamma(0)=x, \dot{\gamma}(0)=V$. Define

$$
\dot{S}(V):=F^{-2}(V) \frac{\mathrm{d}}{\mathrm{d} t}[S(\gamma(t), \dot{\gamma}(t))]_{t=0},
$$

where $S(V)$ denotes the $S$-curvature at $(x, V)$. The weighted Ricci curvature of $(M, F, m)$ is defined by

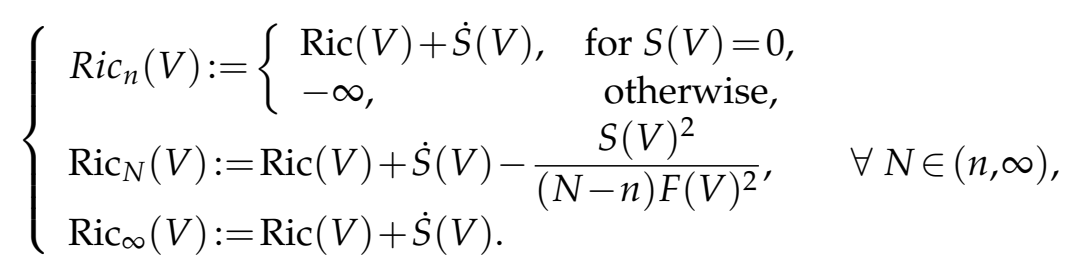

We note that the curvature $\operatorname{Ric}_{N}$ is 0 -homogeneous. 


\subsection{Akbarzadeh's Ricci Tensor, $R i c_{i j}$}

Akbarzadeh's Ricci tensor $\operatorname{Ric}_{i j}$ is defined as follows

$$
\operatorname{Ric}_{i j}:=\frac{\partial^{2}}{\partial y^{i} \partial y^{j}}\left(\frac{F^{2} R i c}{2}\right) \text {. }
$$

We denote second order contravariant tensor of Akbarzadeh's Ricci tensor by $\Re$, that is

$$
\mathfrak{R}:=\operatorname{Ric}^{i j} \frac{\partial}{\partial x^{i}} \otimes \frac{\partial}{\partial x^{j}},
$$

where $R i c^{i j}=g^{i k} g^{j l} R i c_{k l}$. For further details regarding Akbarzadeh's Ricci tensor, see [27].

Similarly, we denote second order contravariant tensor of $h$ by $\mathfrak{h}$, that is

$$
\mathfrak{h}:=h^{i j} \frac{\partial}{\partial x^{i}} \otimes \frac{\partial}{\partial x^{j}}
$$

where $h^{i j}=g^{i k} g^{j l} h_{k l}$.

\subsection{Bochner-Weitzenböck formula}

The following Bochner-Weitzenböck type formula, established by Ohta-Sturm in [22], plays an important role in this paper.

Theorem B. ([22]) (Bochner-Weitzenböck formula) Given $u \in W_{l o c}^{2,2}(M) \cap C^{1}(M)$ with $\Delta_{m} u \in$ $W_{\text {loc }}^{1,2}(M)$, we have

$$
\begin{aligned}
& -\int_{M} D \psi\left(\nabla \nabla u\left(\frac{F^{2}(x, \nabla u)}{2}\right)\right) \mathrm{d} m \\
= & \int_{M} \psi\left\{D\left(\Delta_{m} u\right)(\nabla u)+R i c_{\infty}(\nabla u)+\left|\nabla^{2} u\right|_{H S(\nabla u)}^{2}\right\} \mathrm{d} m
\end{aligned}
$$

for all nonnegative functions $\psi \in W_{c}^{1,2}(M) \cap L^{\infty}(M)$. Given $u \in C^{\infty}(M)$, the pointwise version of the identity is

$$
\Delta_{m}^{\nabla u}\left(\frac{F^{2}(\nabla u)}{2}\right)=D\left(\Delta_{m} u\right)(\nabla u)+\operatorname{Ric}_{\infty}(\nabla u)+\left|\nabla^{2} u\right|_{H S(\nabla u)}^{2} .
$$

Here $\left|\nabla^{2} u\right|_{H S(\nabla u)}^{2}$ denotes the Hilbert-Schmidt norm with respect to $g_{\nabla u}$.

\subsection{Global solutions to $\partial_{t} u=\Delta_{m} u$}

We say that a function $u$ on $[0, T] \times M, T>0$, is a global solution to the nonlinear heat equation $\partial_{t} u=\Delta_{m} u$ if it satisfies the following:

(i) $u(x, t) \in L^{2}\left([0, T], H^{1}(M)\right) \cap H^{1}\left([0, T], H^{-1}(M)\right)$;

(ii) For any test function $\phi \in C^{\infty}(M)$ and for all $t \in[0, T]$,

$$
\int_{M} \phi \partial_{t} u \mathrm{~d} m=-\int_{M} D \phi(\nabla u) \mathrm{d} m \text {. }
$$




\section{Space-time gradient estimates for positive global solutions}

Firstly, we have the following global space-time gradient estimate for the system (1.1)(1.2).

Theorem 3.1. Let $\left(M^{n}, F(t)\right)_{t \in[0, T]}$ be a closed solution to the Finsler-geometric flow (1.2). Assume that there are four positive real numbers $K_{1}, K_{2}, K_{3}$ and $K_{4}$ such that for all $t \in[0, T]$,

$$
\mathfrak{R} \geq-K_{1}, \quad-K_{2} \leq \mathfrak{h} \leq K_{3}, \quad|\nabla \mathfrak{h}| \leq K_{4},
$$

and S-curvature vanishes. Consider a positive global solution $u=u(x, t)$ of the equation (1.1). Let $f=\log u$. Then for any $\alpha>1$, on $M \times(0, T]$, we have

$$
\begin{aligned}
& F^{2}(\nabla(\log u))-\alpha \partial_{t}(\log u) \\
\leq & \frac{n \alpha^{2}}{2(1-2 \varepsilon)}\left\{\frac{(\alpha-1) K_{3}+K_{1}+\varepsilon}{2(1-2 \varepsilon)(\alpha-1)}+\frac{1}{t}+\sqrt{\frac{(1-2 \varepsilon)}{\varepsilon}}\left(K_{2}+K_{3}+\sqrt{\frac{2}{n}} K_{4}\right)\right\} .
\end{aligned}
$$

Here $\varepsilon \in\left(0, \frac{1}{2}\right)$ is an arbitrary constant.

The precise definition of the Finsler measure space, $\mathfrak{R}, \mathfrak{h},|\nabla \mathfrak{h}|$, gradient vector field $\nabla$, Finsler-Laplacian $\Delta_{m}$ and the global solution to the nonlinear heat equation (1.1) have been given in Section 2 above.

Remark 3.1. (1). When $h(t)=-R i c_{i j}(t),(1.2)$ is the Finsler-Ricci flow equation. In this case our results reduce to $[23,24]$. We also see that Theorem 3.1 covers [16, Theorem 6].

(2). The condition $S \equiv 0$ is often required in the study of Finsler-geometric flow. Since the $S$-curvature vanishes for Berwald metrics, our results can be applied to any Finslergeometric flow of Berwald metrics on closed manifolds (for example, see [23,28-30]).

Throughout the rest of these notes, we consider a positive solution $u$ of the nonlinear heat equation (1.1) on a compact Finsler manifold $\left(M^{n}, F, m\right)$. The Laplacian and gradient are all with respect to $V:=\nabla u$ and are valid on $M_{u}:=\{x \in M \mid \nabla u(x) \neq 0\}$. Although Chern connection coefficient $\Gamma_{j k}^{i}(\nabla f)$ is not compatible with respect to $g \nabla f$, it is torsion free. Hence, similar to Riemannian case, for a given time $t$, we can choose a normal coordinate system at a fixed point of $M_{u}$. We will compute at a fixed point and this point we have

$$
h^{i j}(\nabla f)=h_{i j}(\nabla f), \quad\left|\nabla^{2} f\right|_{H S(\nabla f)}^{2}=\sum_{i, j} f_{i j}^{2}, \quad \sum_{i=1}^{n} f_{i i}=\Delta_{m} f, \quad \Gamma_{j k}^{i}(\nabla f)=0 .
$$

Let $u(x, t) \in L^{2}\left([0, T], H^{1}(M)\right) \cap H^{1}\left([0, T], H^{-1}(M)\right)$ be a positive global solution (in the sense of distributions) of the nonlinear heat equation (1.1) under Finsler-geometric flow. 
We consider the function $f:=\log u$ which is $H^{2}$ in space and $C^{1, \alpha}$ in both space and time, then $u=e^{f}$. We have

$$
\partial_{t} f=e^{-f} \partial_{t} u, \quad \nabla f=e^{-f} \nabla u, \quad \Delta_{m} f=e^{-f} \Delta_{m} u-F^{2}(\nabla f) .
$$

Hence $f$ satisfies the following equation,

$$
\partial_{t} f=\Delta_{m} f+F^{2}(\nabla f)
$$

for every $t$ in the weak sense that

$$
\int_{M}\left\{-D \phi(\nabla f)+\phi F^{2}(\nabla f)\right\} \mathrm{d} m=\int_{M} \phi \partial_{t} f \mathrm{~d} m
$$

for each $\phi \in H^{1}(M)$. From (3.3), we have $g_{\nabla f}=g_{\nabla u}$ a.e. on $M_{u}$ and $\Delta_{m} f \in H^{1}(M)$ for each $t$.

To prove Theorem 3.1, we need the following several lemmas. First, we will use the following obvious lemma.

Lemma 3.1. ([25]) Let Ricij be a component of Akbarzadeh's Ricci tensor and Ric be the Ricci curvature on $\left(M^{n}, F, m\right)$. We have

$$
\operatorname{Ric}^{i j}(\nabla f) f_{i} f_{j}=\operatorname{Ric}(\nabla f) .
$$

Proceedingly, we have the following lemma.

Lemma 3.2. Let $h(t)$ is a symmetric $(0,2)$-tensor field on $\left(M^{n}, F(t), m\right)$. We have

$$
\frac{\partial\left(h^{i j}(\nabla f)\right)}{\partial x^{i}} f_{j}=h_{\mid i}^{i j} f_{j}+h_{; k}^{i j} \frac{f_{j}}{F}\left(\nabla^{2} f\right)_{i}^{k},
$$

where $h^{i j}{ }_{i}$ denotes the horizontal covariant derivative of $h^{i j}$ and $h_{; k}^{i j}$ denotes the vertical covariant derivative of $h^{i j}$. In particular,

$$
\begin{aligned}
& \frac{\partial\left(R i c^{i j}(\nabla f)\right)}{\partial x^{i}} f_{j}=R i c_{\mid i}^{i j} f_{j}+\operatorname{Ric}^{i j}{ }_{; k} \frac{f_{j}}{F}\left(\nabla^{2} f\right)_{i}^{k}, \\
& \frac{\partial(H(\nabla f))}{\partial x^{j}} f_{j}=H_{\mid j} f_{j}+H_{; k} \frac{f_{j}}{F}\left(\nabla^{2} f\right)_{j}^{k}, \\
& \frac{\partial\left(g^{i j}(\nabla f)\right)}{\partial x^{i}} f_{j}=0,
\end{aligned}
$$

where Ric $_{i j}$ denotes a component of Akbarzadeh's Ricci tensor and $H=g^{i j}$ Ric $_{i j}$ denotes the scalar curvature. 
Proof. We note that

$$
\begin{aligned}
& h^{i j}=\frac{\delta h^{i j}}{\delta x^{i}}+h^{k j} \Gamma_{k i}^{i}+h^{i k} \Gamma_{k i}^{j}=\frac{\partial h^{i j}}{\partial x^{i}}-N_{i}^{r} \frac{\partial h^{i j}}{\partial y^{r}}+h^{k j} \Gamma_{k i}^{i}+h^{i k} \Gamma_{k i}^{j}, \\
& h_{; i}^{i j}=F \frac{\partial h^{i j}}{\partial y^{i}} .
\end{aligned}
$$

Simple differentiation gives

$$
\begin{aligned}
& \frac{\partial\left(h^{i j}(\nabla f)\right)}{\partial x^{i}}=\frac{\partial h^{i j}}{\partial x^{i}}+\frac{\partial h^{i j}}{\partial y^{k}}(\nabla f)_{x^{i}}^{k} \\
= & h_{\mid i}^{i j}-h^{k j} \Gamma_{k i}^{i}-h^{i k} \Gamma_{k i}^{j}+\frac{\partial h^{i j}}{\partial y^{k}}\left((\nabla f)_{x^{i}}^{k}+N_{i}^{k}\right) \\
= & h_{\mid i}^{i j}-h^{k j} \Gamma_{k i}^{i}-h^{i k} \Gamma_{k i}^{j}+\frac{\partial h^{i j}}{\partial y^{k}}\left(\nabla^{2} f\right)_{i}^{k} .
\end{aligned}
$$

We can choose a normal coordinate system at a fixed point of $M_{u}$. We will compute at a fixed point and at this point we have

$$
\frac{\partial\left(h^{i j}(\nabla f)\right)}{\partial x^{i}} f_{j}=h^{i j} f_{j}+\frac{\partial h^{i j}}{\partial y^{k}}\left(\nabla^{2} f\right)_{i}^{k} f_{j}=h_{\mid i}^{i j} f_{j}+h_{; k}^{i j} \frac{f_{j}}{F}\left(\nabla^{2} f\right)_{i}^{k} .
$$

Eqs. (3.7) and (3.8) are direct consequences of (3.6). Eq. (3.9) is direct consequences of the fact $C_{V}(V, X, Y)=0, g_{\mid s}^{i j}=0$ and $g_{; s}^{i j}=2 C_{i j s}$.

Proceedingly, we have the following lemma.

Lemma 3.3. Let $\left(M^{n}, F(t), m\right)$ be a closed solution to the Finsler-geometric flow (1.2). Then we have

$$
\partial_{t}\left(F^{2}(\nabla f)\right)=-2 h^{i j}(\nabla f) f_{i} f_{j}+2 D\left(\partial_{t} f\right)(\nabla f) .
$$

Proof. Simple differentiation gives

$$
\begin{aligned}
\partial_{t}\left(F^{2}(\nabla f)\right) & =\partial_{t}\left(g^{i j}(\nabla f) f_{i} f_{j}\right) \\
& =2 g^{i j}(\nabla f)\left(\partial_{t} f\right)_{i} f_{j}+\partial_{t}\left(g^{i j}(\nabla f)\right) f_{i} f_{j} \\
& =2 g^{i j}(\nabla f)\left(\partial_{t} f\right)_{i} f_{j}+\partial_{t}\left(g^{i j}\right)(\nabla f) f_{i} f_{j}+\frac{\partial g^{i j}}{\partial y^{k}}\left(\partial_{t} f^{k}\right) f_{i} f_{j} \\
& =-2 h^{i j}(\nabla f) f_{i} f_{j}+2 D\left(\partial_{t} f\right)(\nabla f),
\end{aligned}
$$

where the last equality used $\partial_{t} g^{i j}=-2 h^{i j}$ and $C_{V}(V, X, Y)=0$. 
Let

$$
\mathcal{G}(x, t)=F^{2}(\nabla f)(x, t)-\alpha \partial_{t} f(x, t),
$$

where $\alpha>1$ is a constant. $\mathcal{J}(x, t)=\alpha \partial_{t} f(x, t)$ lies in $H^{1}(M)$ and $\mathcal{G}(x, t)$ lies in $H^{1}(M)$ for each $t$ and is Hölder continuous in both space and time.

Proceeding, we have the following lemma.

Lemma 3.4. In the sense of distributions, $\mathcal{J}(x, t)$ satisfies the parabolic differential equality

$$
\begin{aligned}
& \Delta_{m}^{\nabla f} \mathcal{J}-\partial_{t} \mathcal{J}+2 D \mathcal{J}(\nabla f) \\
= & 2 \alpha h^{i j}(\nabla f) f_{i} f_{j}+2 \alpha h^{i j}(\nabla f) f_{i j}+2 \alpha h_{i j}^{i j} f_{j}+2 \alpha h_{; k}^{i j} \frac{f_{j}}{F}\left(\nabla^{2} f\right)_{i}^{k},
\end{aligned}
$$

where $h^{i j}$ denotes the horizontal covariant derivative of $h^{i j}$ and $h_{; k}^{i j}$ denotes the vertical covariant derivative of $h^{i j}$.

Proof. For any non-negative test function $\phi \in H_{0}^{1}(M \times(0, T))$ whose support is included in the domain of the local coordinate, we have

$$
\begin{aligned}
& \partial_{t}(D(\alpha \phi)(\nabla f)) \\
= & \partial_{t}\left(g^{i j}(\nabla f)(\alpha \phi)_{i} f_{j}\right) \\
= & \partial_{t}\left(g^{i j}(\nabla f)\right)(\alpha \phi)_{i} f_{j}+g^{i j}(\nabla f)\left(\partial_{t}(\alpha \phi)\right)_{i} f_{j}+g^{i j}(\nabla f)(\alpha \phi)_{i}\left(\partial_{t} f\right)_{j} \\
= & \partial_{t}\left(g^{i j}\right)(\nabla f)(\alpha \phi)_{i} f_{j}+\frac{\partial g^{i j}}{\partial y^{k}}\left(\partial_{t} f^{k}\right)(\alpha \phi)_{i} f_{j}+g^{i j}(\nabla f)\left(\partial_{t}(\alpha \phi)\right)_{i} f_{j}+g^{i j}(\nabla f)(\alpha \phi)_{i}\left(\partial_{t} f\right)_{j} \\
= & -2 h^{i j}(\nabla f)(\alpha \phi)_{i} f_{j}+D\left(\partial_{t}(\alpha \phi)\right)(\nabla f)+D(\alpha \phi)\left(\nabla^{\nabla f}\left(\partial_{t} f\right)\right),
\end{aligned}
$$

where the last equality used $\partial_{t} g^{i j}=-2 h^{i j}$ and $C_{V}(V, X, Y)=0$. That is,

$$
\begin{aligned}
& -D(\alpha \phi)\left(\nabla^{\nabla f}\left(\partial_{t} f\right)\right) \\
= & -\partial_{t}(D(\alpha \phi)(\nabla f))+D\left(\partial_{t}(\alpha \phi)\right)(\nabla f)-2 h^{i j}(\nabla f) \frac{\partial(\alpha \phi)}{\partial x^{i}} \frac{\partial f}{\partial x^{j}} .
\end{aligned}
$$

Multiplying the LHS of (3.13) by $\phi$, integrating and then substituting (3.14), we get

$$
\begin{gathered}
\mathcal{A}=\int_{0}^{T} \int_{M}\left\{-D \phi\left(\nabla^{\nabla f} \mathcal{J}\right)+\mathcal{J} \partial_{t} \phi+2 \phi D \mathcal{J}(\nabla f)\right\} \mathrm{d} m \mathrm{~d} t \\
=\int_{0}^{T} \int_{M}\left\{-D(\alpha \phi)\left(\nabla^{\nabla f}\left(\partial_{t} f\right)\right)+\partial_{t}(\phi \alpha) \partial_{t} f+2 \alpha \phi D\left(\partial_{t} f\right)(\nabla f)\right\} \mathrm{d} m \mathrm{~d} t \\
=\int_{0}^{T} \int_{M}\left\{-\partial_{t}(D(\alpha \phi)(\nabla f))+D\left(\partial_{t}(\alpha \phi)\right)(\nabla f)-2 h^{i j}(\nabla f) \frac{\partial(\alpha \phi)}{\partial x^{i}} \frac{\partial f}{\partial x^{j}}\right. \\
\left.\quad+\partial_{t}(\phi \alpha)\left(\Delta_{m} f+F^{2}(\nabla f)\right)+2 \alpha \phi D\left(\partial_{t} f\right)(\nabla f)\right\} \mathrm{d} m \mathrm{~d} t .
\end{gathered}
$$


Using Lemma 3.3 and the fact that

$$
\int_{0}^{T} \int_{M} \partial_{t}(D(\alpha \phi)(\nabla f)) \mathrm{d} m \mathrm{~d} t=0
$$

we arrive at

$$
\begin{aligned}
\mathcal{A}= & \int_{0}^{T} \int_{M}\left\{-\partial_{t}(D(\alpha \phi)(\nabla f))+D\left(\partial_{t}(\alpha \phi)\right)(\nabla f)-2 h^{i j}(\nabla f) \frac{\partial(\alpha \phi)}{\partial x^{i}} \frac{\partial f}{\partial x^{j}}\right. \\
& \left.+\partial_{t}(\phi \alpha)\left(\Delta_{m} f+F^{2}(\nabla f)\right)+2 \alpha \phi D\left(\partial_{t} f\right)(\nabla f)\right\} \mathrm{d} m \mathrm{~d} t \\
= & \int_{0}^{T} \int_{M}\left\{-2 h^{i j}(\nabla f) \frac{\partial(\alpha \phi)}{\partial x^{i}} \frac{\partial f}{\partial x^{j}}+\partial_{t}(\phi \alpha)\left(F^{2}(\nabla f)\right)+2 \alpha \phi D\left(\partial_{t} f\right)(\nabla f)\right\} \mathrm{d} m \mathrm{~d} t \\
= & \int_{0}^{T} \int_{M}\left\{-2 h^{i j}(\nabla f) \frac{\partial(\alpha \phi)}{\partial x^{i}} \frac{\partial f}{\partial x^{j}}+\partial_{t}(\phi \alpha)\left(F^{2}(\nabla f)\right)+\alpha \phi \partial_{t}\left(F^{2}(\nabla f)\right)\right. \\
& \left.\quad+2 \alpha \phi h^{i j}(\nabla f) f_{i} f_{j}\right\} \mathrm{d} m \mathrm{~d} t \\
= & \int_{0}^{T} \int_{M} \phi\left\{2 \alpha h^{i j}(\nabla f) f_{i} f_{j}+2 \alpha h^{i j}(\nabla f) f_{i j}+2 \alpha \frac{\partial\left(h^{i j}(\nabla f)\right)}{\partial x^{i}} f_{j}\right\} \mathrm{d} m \mathrm{~d} t .
\end{aligned}
$$

From (3.6) we have

$$
\mathcal{A}=\int_{0}^{T} \int_{M} \phi\left\{2 \alpha h^{i j}(\nabla f) f_{i} f_{j}+2 \alpha h^{i j}(\nabla f) f_{i j}+2 \alpha h^{i j} f_{j}+2 \alpha h_{; k}^{i j} \frac{f_{j}}{F}\left(\nabla^{2} f\right)_{i}^{k}\right\} \mathrm{d} m \mathrm{~d} t .
$$

This completes the proof of the lemma.

Now we can compute a parabolic partial differential equality for $\mathcal{G}(x, t)$ which will be the key to the proof of our Theorem 3.1.

Lemma 3.5. In the sense of distributions, $\mathcal{G}(x, t)$ satisfies the parabolic differential equality

$$
\Delta_{m}^{\nabla f} \mathcal{G}-\partial_{t} \mathcal{G}+2 D \mathcal{G}(\nabla f)=\mathcal{B}
$$

where

$$
\begin{gathered}
\mathcal{B}(x, t)=-2(\alpha-1) h^{i j}(\nabla f) f_{i} f_{j}-2 \alpha h^{i j}(\nabla f) f_{i j}-2 \alpha h_{\mid i}^{i j} f_{j}-2 \alpha h_{; k}^{i j} \frac{f_{j}}{F}\left(\nabla^{2} f\right)_{i}^{k} \\
+2 R i c(\nabla f)+2\left|\nabla^{2} f\right|_{H S(\nabla f)}^{2} .
\end{gathered}
$$

Proof. For any non-negative test function $\phi \in H_{0}^{1}(M \times(0, T))$, we compute

$$
\begin{aligned}
& \int_{0}^{T} \int_{M}\left\{-D \phi\left(\nabla^{\nabla f} \mathcal{G}\right)+\mathcal{G} \partial_{t} \phi+2 \phi D \mathcal{G}(\nabla f)\right\} \mathrm{d} m \mathrm{~d} t \\
= & -\mathcal{A}+\int_{0}^{T} \int_{M}\left\{-D \phi\left(\nabla^{\nabla f}\left(F^{2}(\nabla f)\right)\right)+F^{2}(\nabla f) \partial_{t} \phi+2 \phi D\left(F^{2}(\nabla f)\right)(\nabla f)\right\} \mathrm{d} m \mathrm{~d} t
\end{aligned}
$$




$$
\begin{aligned}
=-\mathcal{A} & +\int_{0}^{T} \int_{M}\left\{-D \phi\left(\nabla^{\nabla f}\left(F^{2}(\nabla f)\right)\right)-\phi \partial_{t}\left(F^{2}(\nabla f)\right)+2 \phi D\left(F^{2}(\nabla f)\right)(\nabla f)\right\} \mathrm{d} m \mathrm{~d} t \\
=-\mathcal{A} & +\int_{0}^{T} \int_{M}\left\{-D \phi\left(\nabla^{\nabla f}\left(F^{2}(\nabla f)\right)\right)-2 \phi D\left(\partial_{t} f\right)(\nabla f)+2 \phi h^{i j}(\nabla f) f_{i} f_{j}\right. \\
& \left.+2 \phi D\left(F^{2}(\nabla f)\right)(\nabla f)\right\} \mathrm{d} m \mathrm{~d} t \\
=-\mathcal{A} & +\int_{0}^{T} \int_{M}\left\{-D \phi\left(\nabla^{\nabla f}\left(F^{2}(\nabla f)\right)\right)-2 \phi D\left(\Delta_{m} f\right)(\nabla f)-2 \phi D\left(F^{2}(\nabla f)\right)(\nabla f)\right. \\
& \left.\quad+2 \phi h^{i j}(\nabla f) f_{i} f_{j}+2 \phi D\left(F^{2}(\nabla f)\right)(\nabla f)\right\} \mathrm{d} m \mathrm{~d} t \\
=-\mathcal{A} & +\int_{0}^{T} \int_{M}\left\{-D \phi\left(\nabla^{\nabla f}\left(F^{2}(\nabla f)\right)\right)-2 \phi D\left(\Delta_{m} f\right)(\nabla f)+2 \phi h^{i j}(\nabla f) f_{i} f_{j}\right\} \mathrm{d} m \mathrm{~d} t .
\end{aligned}
$$

By applying the Bochner-Weitzenböck formula (2.10) and noticing that $S=0$ implies $\operatorname{Ric}_{\infty}(V)=\operatorname{Ric}(V)$, we have

$$
\begin{aligned}
& -\mathcal{A}+\int_{0}^{T} \int_{M}\left\{-D \phi\left(\nabla^{\nabla f}\left(F^{2}(\nabla f)\right)\right)-2 \phi D\left(\Delta_{m} f\right)(\nabla f)+2 \phi h^{i j}(\nabla f) f_{i} f_{j}\right\} \mathrm{d} m \mathrm{~d} t \\
= & -\mathcal{A}+\int_{0}^{T} \int_{M}\left\{2 \phi R i c(\nabla f)+2 \phi\left|\nabla^{2} f\right|_{H S(\nabla f)}^{2}+2 \phi h^{i j}(\nabla f) f_{i} f_{j}\right\} \mathrm{d} m \mathrm{~d} t .
\end{aligned}
$$

Now, substituting $\mathcal{A}$ from (3.16), we have

$$
\begin{aligned}
\mathcal{B}(x, t)= & -2(\alpha-1) h^{i j}(\nabla f) f_{i} f_{j}-2 \alpha h^{i j}(\nabla f) f_{i j}-2 \alpha h_{\mid i}^{i j} f_{j}-2 \alpha h_{; k}^{i j} \frac{f_{j}}{F}\left(\nabla^{2} f\right)_{i}^{k} \\
& +2 \operatorname{Ric}(\nabla f)+2\left|\nabla^{2} f\right|_{H S(\nabla f)}^{2} .
\end{aligned}
$$

Now we have all the ingredients that we need to complete the proof of Theorem 3.1.

Proof of Theorem 3.1. By our assumption and (3.17), we have

$$
\begin{aligned}
& \Delta_{m}^{\nabla f} \mathcal{G}-\partial_{t} \mathcal{G}+2 D \mathcal{G}(\nabla f) \\
&=-2(\alpha-1) h^{i j}(\nabla f) f_{i} f_{j}-2 \alpha h^{i j}(\nabla f) f_{i j}-2 \alpha h^{i j} f_{j}-2 \alpha h_{; k}^{i j} \frac{f_{j}}{F}\left(\nabla^{2} f\right)_{i}^{k} \quad+2 R i c(\nabla f)+2\left|\nabla^{2} f\right|_{H S(\nabla f)}^{2} \\
& \geq-2(\alpha-1) K_{3} F^{2}(\nabla f)-2 \varepsilon f_{i j}^{2}-\frac{n \alpha^{2}}{2 \varepsilon}\left(K_{2}+K_{3}\right)^{2}-2 \varepsilon F^{2}(\nabla f)-\frac{\alpha^{2}}{2 \varepsilon} K_{4}^{2} \\
& \quad-2 \varepsilon f_{k i}^{2}-\frac{\alpha^{2}}{2 \varepsilon} K_{4}^{2}-2 K_{1} F^{2}(\nabla f)+2 f_{i j}^{2} \\
&=2(1-2 \varepsilon) f_{i j}^{2}-2\left((\alpha-1) K_{3}+K_{1}+\varepsilon\right) F^{2}(\nabla f)-\frac{n \alpha^{2}}{2 \varepsilon}\left(K_{2}+K_{3}\right)^{2}-\frac{\alpha^{2}}{\varepsilon} K_{4}^{2} \\
& \geq \frac{2(1-2 \varepsilon)}{n}\left(\Delta_{m} f\right)^{2}-2\left((\alpha-1) K_{3}+K_{1}+\varepsilon\right) F^{2}(\nabla f)-\frac{\alpha^{2}}{\varepsilon}\left(\frac{n}{2}\left(K_{2}+K_{3}\right)^{2}+K_{4}^{2}\right),
\end{aligned}
$$


where we used

$$
\begin{gathered}
-2 \alpha h_{i j} f_{i j} \geq-2 \varepsilon \sum_{i, j} f_{i j}^{2}-\frac{\alpha^{2}}{2 \varepsilon} \sum_{i, j} h_{i j}^{2} \geq-2 \varepsilon \sum_{i, j} f_{i j}^{2}-\frac{n \alpha^{2}}{2 \varepsilon}\left(K_{2}+K_{3}\right)^{2}, \\
-2 \alpha h_{\mid i}^{i j} f_{j} \geq-2 \varepsilon F^{2}(\nabla f)-\frac{\alpha^{2}}{2 \varepsilon} \sum_{i, j}\left(h^{i j}\right)^{2} \geq-2 \varepsilon F^{2}(\nabla f)-\frac{\alpha^{2}}{2 \varepsilon} K_{4}^{2}, \\
-2 \alpha h_{; k}^{i j} \frac{f_{j}}{F}\left(\nabla^{2} f\right)_{i}^{k} \geq-2 \varepsilon \sum_{i, k} f_{i k}^{2}-\frac{\alpha^{2}}{2 \varepsilon} \sum_{i, k}\left(\sum_{j} h_{; j}^{i j} \frac{f_{j}}{F}\right)^{2} \geq-2 \varepsilon \sum_{i, k} f_{i k}^{2}-\frac{\alpha^{2}}{2 \varepsilon} \sum_{i, j, k}\left(h_{; j k}^{i j}\right)^{2} \\
\geq-2 \varepsilon \sum_{i, k} f_{i k}^{2}-\frac{\alpha^{2}}{2 \varepsilon} K_{4}^{2}
\end{gathered}
$$

and the Cauchy inequality

$$
\sum_{i, j} f_{i j}^{2} \geq \frac{1}{n}\left(\Delta_{m} f\right)^{2}
$$

Noticing that

$$
\Delta_{m} f=-F^{2}(\nabla f)+\partial_{t} f=-\frac{1}{\alpha}\left(\mathcal{G}+(\alpha-1) F^{2}(\nabla f)\right),
$$

hence (3.18) shows

$$
\begin{aligned}
& \Delta_{m}^{\nabla f} \mathcal{G}-\partial_{t} \mathcal{G}+2 D \mathcal{G}(\nabla f) \\
\geq & \frac{2(1-2 \varepsilon)}{n \alpha^{2}}\left(\mathcal{G}+(\alpha-1) F^{2}(\nabla f)\right)^{2}-2\left((\alpha-1) K_{3}+K_{1}+\varepsilon\right) F^{2}(\nabla f)-\frac{\alpha^{2}}{\varepsilon}\left(\frac{n}{2}\left(K_{2}+K_{3}\right)^{2}+K_{4}^{2}\right) .
\end{aligned}
$$

Define $\overline{\mathcal{G}}=t \mathcal{G}$. Then

$$
\begin{aligned}
& \Delta_{m}^{\nabla f} \overline{\mathcal{G}}-\partial_{t} \overline{\mathcal{G}}=t\left(\Delta_{m}^{\nabla f} \mathcal{G}-\partial_{t} \mathcal{G}\right)-\frac{\overline{\mathcal{G}}}{t} \\
& \geq t\left\{\frac{2(1-2 \varepsilon)}{n \alpha^{2}}\left(\mathcal{G}+(\alpha-1) F^{2}(\nabla f)\right)^{2}-2\left((\alpha-1) K_{3}+K_{1}+\varepsilon\right) F^{2}(\nabla f)\right. \\
&\left.\quad-\frac{\alpha^{2}}{\varepsilon}\left(\frac{n}{2}\left(K_{2}+K_{3}\right)^{2}+K_{4}^{2}\right)-2 D \mathcal{G}(\nabla f)\right\}-\frac{\overline{\mathcal{G}}}{t} \\
&=\frac{2(1-2 \varepsilon) t}{n \alpha^{2}}\left(\mathcal{G}+(\alpha-1) F^{2}(\nabla f)\right)^{2}-2\left((\alpha-1) K_{3}+K_{1}+\varepsilon\right) t F^{2}(\nabla f) \\
& \quad-\frac{\alpha^{2} t}{\varepsilon}\left(\frac{n}{2}\left(K_{2}+K_{3}\right)^{2}+K_{4}^{2}\right)-2 D \overline{\mathcal{G}}(\nabla f)-\frac{\overline{\mathcal{G}}}{t} .
\end{aligned}
$$

Let $\frac{F^{2}(\nabla f)}{\mathcal{G}}=\mu$. Then we have $\mu \geq 0$ (otherwise the assertion of the theorem is trivial) and

$$
\Delta_{m}^{\nabla f} \overline{\mathcal{G}}-\partial_{t} \overline{\mathcal{G}}+2 D \overline{\mathcal{G}}(\nabla f)
$$




$$
\begin{aligned}
& \geq \frac{2(1-2 \varepsilon) t}{n \alpha^{2}}\left(\mathcal{G}+(\alpha-1) F^{2}(\nabla f)\right)^{2}-2\left((\alpha-1) K_{3}+K_{1}+\varepsilon\right) t F^{2}(\nabla f) \\
& \quad-\frac{\alpha^{2} t}{\varepsilon}\left(\frac{n}{2}\left(K_{2}+K_{3}\right)^{2}+K_{4}^{2}\right)-\frac{\overline{\mathcal{G}}}{t} \\
& =\frac{2(1-2 \varepsilon)}{n \alpha^{2} t}(1+(\alpha-1) \mu)^{2} \overline{\mathcal{G}}^{2}-\left(2\left((\alpha-1) K_{3}+K_{1}+\varepsilon\right) \mu+\frac{1}{t}\right) \overline{\mathcal{G}} \\
& \quad-\frac{\alpha^{2} t}{\varepsilon}\left(\frac{n}{2}\left(K_{2}+K_{3}\right)^{2}+K_{4}^{2}\right):=\mathcal{D} .
\end{aligned}
$$

Fix arbitrary $t \in(0, T]$ and assume that $\overline{\mathcal{G}}$ achieves its maximum at the point $\left(x_{0}, t_{0}\right) \in$ $M \times[0, t]$ and $\overline{\mathcal{G}}\left(x_{0}, t_{0}\right)>0$ (otherwise the proof is trivial), which implies $t_{0}>0$. We shall show $\mathcal{D}\left(x_{0}, t_{0}\right) \leq 0$. Assume the contrary, $\mathcal{D}\left(x_{0}, t_{0}\right)>0$. It would imply $\mathcal{D}>0$ on a neighborhood of $\left(x_{0}, t_{0}\right)$. Hence, according to (3.21) on such a neighborhood, the function $\overline{\mathcal{G}}$ would be a strict sub-solution to the linear parabolic operator

$$
\Delta_{m}^{\nabla f} \overline{\mathcal{G}}-\partial_{t} \overline{\mathcal{G}}+2 D \overline{\mathcal{G}}(\nabla f) .
$$

Therefore, $\overline{\mathcal{G}}\left(x_{0}, t_{0}\right)$ would be strictly less than the supremum of $\overline{\mathcal{G}}$ on the boundary of any small parabolic cylinder $\left[t_{0}-\delta, t_{0}\right] \times B_{\delta}\left(x_{0}\right)$, where $B_{\delta}\left(x_{0}\right):=\left\{y \in M \mid d\left(x_{0}, y\right)<\delta\right\}$. In particular, $\overline{\mathcal{G}}$ could not be maximal at $\left(x_{0}, t_{0}\right)$, which is a contradiction. Hence, $\mathcal{D}\left(x_{0}, t_{0}\right) \leq$ 0 , that is at $\left(x_{0}, t_{0}\right)$

$$
\begin{gathered}
0 \geq \frac{2(1-2 \varepsilon)}{n \alpha^{2} t}(1+(\alpha-1) \mu)^{2} \overline{\mathcal{G}}^{2}-\left(2\left((\alpha-1) K_{3}+K_{1}+\varepsilon\right) \mu+\frac{1}{t}\right) \overline{\mathcal{G}} \\
-\frac{\alpha^{2} t}{\varepsilon}\left(\frac{n}{2}\left(K_{2}+K_{3}\right)^{2}+K_{4}^{2}\right) .
\end{gathered}
$$

All the following computations are at the point $\left(x_{0}, t_{0}\right)$. Noticing that

$$
\begin{aligned}
& \frac{\mu}{(1+(\alpha-1) \mu)^{2}}=\frac{1}{\alpha-1} \frac{(\alpha-1) \mu}{(1+(\alpha-1) \mu)^{2}} \leq \frac{1}{4(\alpha-1)}, \\
& \frac{1}{(1+(\alpha-1) \mu)^{2}} \leq 1 .
\end{aligned}
$$

Solving the quadratic inequality of $\overline{\mathcal{G}}$ in (3.22) yields

$$
\begin{aligned}
\overline{\mathcal{G}} & \leq \frac{n \alpha^{2} t_{0}}{4(1-2 \varepsilon)(1+(\alpha-1) \mu)^{2}}\left\{\left(2\left((\alpha-1) K_{3}+K_{1}+\varepsilon\right) \mu+\frac{1}{t_{0}}\right)\right. \\
& \left.+\sqrt{\left(2\left((\alpha-1) K_{3}+K_{1}+\varepsilon\right) \mu+\frac{1}{t_{0}}\right)^{2}+\frac{8(1-2 \varepsilon)}{n \varepsilon}(1+(\alpha-1) \mu)^{2}\left(\frac{n}{2}\left(K_{2}+K_{3}\right)^{2}+K_{4}^{2}\right)}\right\} \\
& \leq \frac{n \alpha^{2} t_{0}}{4(1-2 \varepsilon)(1+(\alpha-1) \mu)^{2}}\left\{2\left(2\left((\alpha-1) K_{3}+K_{1}+\varepsilon\right) \mu+\frac{1}{t_{0}}\right)\right.
\end{aligned}
$$




$$
\begin{gathered}
\left.+2 \sqrt{\frac{2(1-2 \varepsilon)}{n \varepsilon}}(1+(\alpha-1) \mu)\left(\sqrt{\frac{n}{2}}\left(K_{2}+K_{3}\right)+K_{4}\right)\right\} \\
\leq \frac{n \alpha^{2} t_{0}}{2(1-2 \varepsilon)}\left\{\frac{(\alpha-1) K_{3}+K_{1}+\varepsilon}{2(\alpha-1)}+\frac{1}{t_{0}}+\sqrt{\frac{1-2 \varepsilon}{\varepsilon}}\left(K_{2}+K_{3}+\sqrt{\frac{2}{n}} K_{4}\right)\right\} \\
\leq \frac{n \alpha^{2} t_{0}}{2(1-2 \varepsilon)}\left\{\frac{(\alpha-1) K_{3}+K_{1}+\varepsilon}{2(\alpha-1)(1-2 \varepsilon)}+\frac{1}{t_{0}}+\sqrt{\frac{1-2 \varepsilon}{\varepsilon}}\left(K_{2}+K_{3}+\sqrt{\frac{2}{n}} K_{4}\right)\right\} .
\end{gathered}
$$

Since $t \geq t_{0}$, we have

$$
\begin{aligned}
& \overline{\mathcal{G}}(x, t) \leq \overline{\mathcal{G}}\left(x_{0}, t_{0}\right) \\
\leq & \frac{n \alpha^{2} t}{2(1-2 \varepsilon)}\left\{\frac{(\alpha-1) K_{3}+K_{1}+\varepsilon}{2(\alpha-1)(1-2 \varepsilon)}+\frac{1}{t}+\sqrt{\frac{1-2 \varepsilon}{\varepsilon}}\left(K_{2}+K_{3}+\sqrt{\frac{2}{n}} K_{4}\right)\right\}
\end{aligned}
$$

and for all $x \in M$, it holds that

$$
\mathcal{G}(x, t) \leq \frac{n \alpha^{2}}{2(1-2 \varepsilon)}\left\{\frac{(\alpha-1) K_{3}+K_{1}+\varepsilon}{2(\alpha-1)(1-2 \varepsilon)}+\frac{1}{t}+\sqrt{\frac{1-2 \varepsilon}{\varepsilon}}\left(K_{2}+K_{3}+\sqrt{\frac{2}{n}} K_{4}\right)\right\} .
$$

Since $t$ is arbitrary in $t \in[0, T]$, we obtain (3.1). Hence, we complete the proof.

Similar to [16, Corollary 8], integrating the gradient estimate in space-time as in [3], we can derive the following parabolic Harnack type inequality.

Corollary 3.1. Let $\left(M^{n}, F(t)\right)_{t \in[0, T]}$ be a closed solution to the Finsler-geometric flow (1.2). Assume that there are four positive real numbers $K_{1}, K_{2}, K_{3}$ and $K_{4}$ such that for all $t \in[0, T]$,

$$
\mathfrak{R} \geq-K_{1}, \quad-K_{2} \leq \mathfrak{h} \leq K_{3}, \quad|\nabla \mathfrak{h}| \leq K_{4},
$$

and S-curvature vanishes. Consider a positive global solution $u=u(x, t)$ of the equation (1.1). Let $f=\log u$. Then for $\left(x_{1}, t_{1}\right) \in M^{n} \times(0, T)$ and $\left(x_{2}, t_{2}\right) \in M^{n} \times(0, T)$ such that $t_{1}<t_{2}$, we have

$$
\begin{aligned}
& u\left(x_{1}, t_{1}\right) \leq u\left(x_{2}, t_{2}\right)\left(\frac{t_{2}}{t_{1}}\right)^{n \alpha} \exp \left\{\int_{0}^{1} \frac{\alpha}{4} \frac{\left.F(\dot{\eta}(s))^{2}\right|_{\tau}}{t_{2}-t_{1}} \mathrm{~d} s\right. \\
& \left.\quad+n \alpha\left(\frac{(\alpha-1) K_{3}+K_{1}+\frac{1}{4}}{\alpha-1}+\sqrt{2}\left(K_{2}+K_{3}+\sqrt{\frac{2}{n}} K_{4}\right)\right)\left(t_{2}-t_{1}\right)\right\},
\end{aligned}
$$

whenever $\alpha>1$. Here $\eta(s)$ be a smooth curve connecting $x$ and $y$ with $\eta(1)=x$ and $\eta(0)=y$, and $\left.F(\dot{\eta}(s))\right|_{\tau}$ is the length of the vector $\dot{\eta}(s)$ at time $\tau(s)=(1-s) t_{2}+s t_{1}$. 
Proof. Let $\eta(s)$ be a smooth curve connecting $x$ and $y$ with $\eta(1)=x$ and $\eta(0)=y$, and $\left.F(\dot{\eta}(s))\right|_{\tau}$ is the length of the vector $\dot{\eta}(s)$ at time $\tau(s)=(1-s) t_{2}+s t_{1}$. Choosing $\varepsilon=\frac{1}{4}$ in (3.1) gives

$$
-\partial_{t} f \leq n \alpha\left\{\frac{(\alpha-1) K_{3}+K_{1}+\frac{1}{4}}{\alpha-1}+\frac{1}{t}+\sqrt{2}\left(K_{2}+K_{3}+\sqrt{\frac{2}{n}} K_{4}\right)\right\}-\frac{1}{\alpha} F^{2}(\nabla f) .
$$

Let $l(s)=\log u(\eta(s), \tau(s))=f(\eta(s), \tau(s))$. Then

$$
\begin{aligned}
& f\left(x_{1}, t_{1}\right)-f\left(x_{2}, t_{2}\right)=\int_{0}^{1} \frac{\mathrm{d}}{\mathrm{d} s}(f(\eta(s), \tau(s))) \mathrm{d} s=\int_{0}^{1}\left(t_{2}-t_{1}\right)\left(\frac{D f(\dot{\eta}(s))}{t_{2}-t_{1}}-\partial_{t} f\right) \mathrm{d} s \\
\leq & \int_{0}^{1}\left(t_{2}-t_{1}\right)\left\{\frac{F(\dot{\eta}(s)) F(\nabla f)}{t_{2}-t_{1}}-\partial_{t} f\right\} \mathrm{d} s \\
\leq & \int_{0}^{1}\left(t_{2}-t_{1}\right)\left\{\frac{F(\dot{\eta}(s)) F(\nabla f)}{t_{2}-t_{1}}+n \alpha\left(\frac{(\alpha-1) K_{3}+K_{1}+\frac{1}{4}}{\alpha-1}+\frac{1}{\tau}\right.\right. \\
& \left.\left.\quad+\sqrt{2}\left(K_{2}+K_{3}+\sqrt{\frac{2}{n}} K_{4}\right)\right)-\frac{1}{\alpha} F^{2}(\nabla f)\right\} \mathrm{d} s \\
\leq & \int_{0}^{1}\left\{\frac{\alpha}{4} \frac{\left.F(\dot{\eta}(s))^{2}\right|_{\tau}}{t_{2}-t_{1}}+\left(t_{2}-t_{1}\right) n \alpha\left(\frac{(\alpha-1) K_{3}+K_{1}+\frac{1}{4}}{\alpha-1}+\frac{1}{\tau}+\sqrt{2}\left(K_{2}+K_{3}+\sqrt{\frac{2}{n}} K_{4}\right)\right)\right\} \mathrm{d} s,
\end{aligned}
$$

where the last inequality used $-A x^{2}+B x \leq \frac{B^{2}}{4 A}$. Using (3.26), we derive

$$
\begin{aligned}
& \log \left(\frac{u\left(x_{1}, t_{1}\right)}{u\left(x_{2}, t_{2}\right)}\right)=f\left(x_{1}, t_{1}\right)-f\left(x_{2}, t_{2}\right) \\
& \leq \int_{0}^{1} \frac{\alpha}{4} \frac{\left.F(\dot{\eta}(s))^{2}\right|_{\tau}}{t_{2}-t_{1}} \mathrm{~d} s+n \alpha\left(\frac{(\alpha-1) K_{3}+K_{1}+\frac{1}{4}}{\alpha-1}+\sqrt{2}\left(K_{2}+K_{3}+\sqrt{\frac{2}{n}} K_{4}\right)\right)\left(t_{2}-t_{1}\right) \\
& \quad+n \alpha \log \left(\frac{t_{2}}{t_{1}}\right) .
\end{aligned}
$$

Therefore, we arrive at

$$
\begin{aligned}
u\left(x_{1}, t_{1}\right) \leq u\left(x_{2}, t_{2}\right) & \left(\frac{t_{2}}{t_{1}}\right)^{n \alpha} \exp \left\{\int_{0}^{1} \frac{\alpha}{4} \frac{\left.F(\dot{\eta}(s))^{2}\right|_{\tau}}{t_{2}-t_{1}} \mathrm{~d} s\right. \\
& \left.+n \alpha\left(\frac{(\alpha-1) K_{3}+K_{1}+\frac{1}{4}}{\alpha-1}+\sqrt{2}\left(K_{2}+K_{3}+\sqrt{\frac{2}{n}} K_{4}\right)\right)\left(t_{2}-t_{1}\right)\right\} .
\end{aligned}
$$




\section{Applications to Finsler-Ricci flow and Finsler-Yamabe flow}

In this section, we apply our results to the special cases of the Finsler-Ricci flow and Finsler-Yamabe flow.

\subsection{The Finsler-Ricci flow}

When $h(t)=-R i c_{i j}(t),(1.2)$ is the Finsler-Ricci flow equation (see $\left.[1,28,31]\right)$. In this situation our results reduced to those in $[23,24]$. Comparing with the Riemannian case, in this case our results in Section 3 need the assumption $|\nabla \mathfrak{R}| \leq K_{4}$. Since the solutions of the nonlinear heat equation (1.1) are lack of higher order regularity, we have to compute $\partial_{t}\left(\Delta_{m} f\right)$ in a weak sense, which produces $R i c_{\mid i}^{i j}$ and $R i c_{; k}^{i j}$. In order to obtain the gradient estimate, we require $|\nabla \Re|$ bounded above.

It is worth to notice that the inequality (2) in [23] was not completely correct. In fact, due to the proof of Lemma 4.1 in [23], Lakzian thought that

$$
\frac{\partial\left(\operatorname{Ric}^{i j}(\nabla f)\right)}{\partial x^{i}} f_{j}=0
$$

by Euler's theorem. This means that the parabolic differential equality (43) in [23] is lack of $\frac{\partial\left(R i c^{i j}(\nabla f)\right)}{\partial x^{i}} f_{j}$. However, we compute

$$
\frac{\partial\left(\operatorname{Ric}^{i j}(\nabla f)\right)}{\partial x^{i}} f_{j}=\operatorname{Ric}^{i j} f_{j}+\operatorname{Ric}_{; k}^{i j} \frac{f_{j}}{F}\left(\nabla^{2} f\right)_{i}^{k} \neq 0,
$$

hence our results generalize and correct the work of Lakzian in [23].

\subsection{The Finsler-Yamabe flow}

When $h(t)=-\frac{1}{2}(H g)(t),(1.2)$ is the Finsler-Yamabe flow equation

$$
\frac{\partial}{\partial t} g(t)=-(H g)(t)
$$

where $H=g^{i j} \operatorname{Ric}_{i j}$ is called the scalar curvature. For further details regarding the FinslerYamabe flow, see [29,32].

However, to prove our Theorem 4.1, it is enough to consider for all $t \in[0, T]$,

$$
\mathfrak{R} \geq-K_{1}, \quad-K_{2} \leq H \leq K_{3}, \quad|\nabla H| \leq K_{4},
$$

and $S$-curvature vanishes to derive the following space-time gradient estimate for the system (1.1)-(4.1). 
Theorem 4.1. Let $\left(M^{n}, F(t)\right)_{t \in[0, T]}$ be a closed solution to the Finsler-Yamabe flow (4.1). Assume that there are four positive real numbers $K_{1}, K_{2}, K_{3}$ and $K_{4}$ such that for all $t \in[0, T]$,

$$
\mathfrak{R} \geq-K_{1}, \quad-K_{2} \leq H \leq K_{3}, \quad|\nabla H| \leq K_{4},
$$

and S-curvature vanishes. Consider a positive global solution $u=u(x, t)$ of the equation (1.1). Let $f=\log u$. Then for any $\alpha>1$, on $M \times(0, T]$, we have

$$
\begin{aligned}
& F^{2}(\nabla(\log u))-\alpha \partial_{t}(\log u) \\
\leq & \frac{n \alpha^{2}}{2(1-\varepsilon)}\left\{\frac{(\alpha-1) K_{2}+K_{1}+\varepsilon}{4(1-\varepsilon)(\alpha-1)}+\frac{1}{t}+\sqrt{\frac{(1-\varepsilon)}{2 \varepsilon}}\left(K_{2}+K_{3}+\sqrt{\frac{2}{n}} K_{4}\right)\right\} .
\end{aligned}
$$

Here $\varepsilon \in(0,1)$ is an arbitrary constant.

The proof of Theorem 4.1 is similar to the one at Section 3. We only to sketch a very simple outline of the proof. First of all, we have the following several lemmas.

Lemma 4.1. Let $\left(M^{n}, F(t), m\right)$ be a closed solution to the Finsler-Yamabe flow (4.1). Then we have

$$
\partial_{t}\left(F^{2}(\nabla f)\right)=H F^{2}(\nabla f)+2 D\left(\partial_{t} f\right)(\nabla f) .
$$

Lemma 4.2. In the sense of distributions, $\mathcal{J}(x, t)$ satisfies the parabolic differential equality

$$
\begin{gathered}
\Delta_{m}^{\nabla f} \mathcal{J}-\partial_{t} \mathcal{J}+2 D \mathcal{J}(\nabla f)=-\alpha H F^{2}(\nabla f)-\alpha H g^{i j}(\nabla f) f_{i j}-\alpha g^{i j} H_{\mid i} f_{j} \\
-\alpha g^{i j} H_{; k} \frac{f_{j}}{F}\left(\nabla^{2} f\right)_{i}^{k}
\end{gathered}
$$

where $H_{\mid i}$ denotes the horizontal covariant derivative of $H$ and $H_{; k}$ denotes the vertical covariant derivative of $H$.

Lemma 4.3. In the sense of distributions, $\mathcal{G}(x, t)$ satisfies the parabolic differential equality

$$
\Delta_{m}^{\nabla f} \mathcal{G}-\partial_{t} \mathcal{G}+2 D \mathcal{G}(\nabla f)=\mathcal{B},
$$

where

$$
\begin{gathered}
\mathcal{B}(x, t)=(\alpha-1) H F^{2}(\nabla f)+\alpha H g^{i j} f_{i j}+\alpha g^{i j} H_{\mid i} f_{j}+\alpha g^{i j} H_{; k} \frac{f_{j}}{F}\left(\nabla^{2} f\right)_{i}^{k} \\
+2 R i c(\nabla f)+2\left|\nabla^{2} f\right|_{H S(\nabla f)}^{2} .
\end{gathered}
$$

Lemma 4.4. In the sense of distributions, $\mathcal{G}(x, t)$ satisfies the parabolic differential inequality

$$
\begin{aligned}
& \Delta_{m}^{\nabla f} \mathcal{G}-\partial_{t} \mathcal{G}+2 D \mathcal{G}(\nabla f) \\
\geq & \frac{2(1-\varepsilon)}{n}\left(\Delta_{m} f\right)^{2}-\left((\alpha-1) K_{2}+K_{1}+\varepsilon\right) F^{2}(\nabla f)-\frac{\alpha^{2}}{2 \varepsilon}\left(\frac{n}{2}\left(K_{2}+K_{3}\right)^{2}+K_{4}^{2}\right) .
\end{aligned}
$$

Then, using Lemma 4.4, the quadratic formula and the maximum principle, we can complete the proof of Theorem 4.1. 


\section{Acknowledgement}

This work was supported by NSFC 11971415 and Nanhu Scholars Program for Young Scholars of XYNU.

\section{References}

[1] Bao D., On two curvature-driven problems in Riemann-Finsler geometry. Finsler geometry, Sapporo 2005-in memory of Makoto Matsumoto, 19C71, Adv. Stud. Pure Math., 48, Math. Soc. Japan, Tokyo, 2007.

[2] Ohta S., Sturm K., Heat flow on Finsler manifolds. Comm. Pure Appl. Math., 62 (2009), 13861433.

[3] Li P., Yau S.-T., On the parabolic kernel of the Schröinger operator. Acta Math., 156 (1986), 153-201.

[4] Huang G., Huang Z., Li H., Gradient estimates for the porous medium equations on Riemannian manifolds. J. Geom. Anal., 23 (2013), 1851-1875.

[5] Li Y., Li-Yau-Hamilton estimates and Bakry-Emery-Ricci curvature. Nonlinear Anal., 113 (2015), 1-32.

[6] Li J., Gradient estimates and Harnack inequalities for nonlinear parabolic and nonlinear elliptic equations on Riemannian manifolds. J. Funct. Anal., 100 (1991), 233-256.

[7] Li J., Gradient estimate for the heat kernel of a complete Riemannian manifold and its applications. J. Funct. Anal., 97 (1991), 293-310.

[8] Ma B., Huang G., Luo Y., Gradient estimates for a nonlinear elliptic equation on complete Riemannian manifolds. Proc. Amer. Math. Soc., 146 (2018), 4993-5002.

[9] Ma B., Zeng F., Hamilton-Souplet-Zhang's gradient estimates and Liouville theorems for a nonlinear parabolic equation. C. R. Math. Acad. Sci. Paris, 356 (2018), 550-557.

[10] Ma L., Gradient estimates for a simple elliptic equation on complete non-compact Riemannian manifolds. J. Funct. Anal., 241 (2006), 374-382.

[11] Wu J., Elliptic gradient estimates for a weighted heat equation and applications. Math. Z., 280 (2015), 451-468.

[12] Yang Y., Gradient estimates for a nonlinear parabolic equation on Riemannian manifolds. Proc. Amer. Math. Soc., 136 (2008), 4095-4102.

[13] Zeng F., Gradient estimates of a nonlinear elliptic equation for the $V$-Laplacian. Bull. Korean Math. Soc., 56 (2019), 853-865.

[14] Liu S., Gradient estimates for solutions of the heat equation under Ricci flow. Pacific J. Math., 243 (2009), 165-180.

[15] Guenther C., The fundamental solution on manifolds with time-dependent metrics. J. Geom. Anal., 12 (2002), 425-436.

[16] Sun J., Gradient estimates for positive solutions of the heat equation under geometric flow. Pacific J. Math., 253 (2011), 489-510.

[17] Bailesteanu M., Cao X., Pulemotov A., Gradient estimates for the heat equation under the Ricci flow. J. Funct. Anal., 258 (2010), 3517-3542.

[18] Guo H., Ishida M., Harnack estimates for nonlinear backward heat equations in geometric flows. J. Funct. Anal., 267 (2014), 2638-2662.

[19] Li Y., Zhu X., Harnack estimates for a heat-type equation under the Ricci flow. J. Differential Equations, 260 (2016), 3270-3301. 
[20] Zhang Q., Some gradient estimates for the heat equation on domains and for an equation by Perelman. Int. Math. Res. Not., 2006, Art. ID 92314, 39 pp.

[21] Zhao L., Fang S., Gradient estimates for a nonlinear Lichnerowicz equation under general geometric flow on complete noncompact manifolds. Pacific J. Math., 285 (2016), 243-256.

[22] Ohta S., Sturm K., Bochner-Weitzenböck formula and Li-Yau estimates on Finsler manifolds. Adv. Math., 252 (2014), 429-448.

[23] Lakzian S., Differential Harnack estimates for positive solutions to heat equation under Finsler-Ricci flow. Pacific J. Math., 278 (2015), 447-462.

[24] Zeng F., He Q., Gradient estimates for a nonlinear heat equation under the Finsler-Ricci flow. Math. Slovaca, 69 (2019), 409-424.

[25] Bao D., Chern S., Shen Z., An Introduction to Riemannian-Finsler Geometry, Grad. Texts in Math., vol. 200, Springer-Verlag, 2000.

[26] Ohta S., Finsler interpolation inequalities. Calc. Var. Partial Differential Equations, 36 (2009), 211-249.

[27] Bao D., Robles C., Ricci and flag curvatures in Finsler geometry. A sampler of RiemannFinsler geometry, 197C259, Math. Sci. Res. Inst. Publ., 50, Cambridge Univ. Press, Cambridge, 2004.

[28] Azami S., Razavi A., Existence and uniqueness for solutions of Ricci flow on Finsler manifolds. Int. J. Geom. Methods Mod. Phys., 10 (2013), 21 pp.

[29] Azami S., Razavi A., Yamabe flow on Berwald manifolds. Int. J. Geom. Methods Mod. Phys., 12 (2015), $27 \mathrm{pp}$.

[30] Ohta S., Vanishing s-curvature of randers spaces. Differential Geom. Appl., 29 (2011), 174-178.

[31] Bidabad B., Ahmadi M., On quasi-Einstein Finsler spaces. Bull. Iranian Math. Soc., 40 (2014), 921-930.

[32] Bidabad B., Ahmadi M., On complete Finslerian Yamabe solitons. Differential Geom. Appl., 66 (2019), 52-60. 